\title{
Osocimab zur Prävention von venösen Thromboembolien
}

Weitz Jl et al. Effect of Osocimab in Preventing Venous Thromboembolism Among Patients Undergoing Knee Arthroplasty: The FOXTROT Randomized Clinical Trial. JAMA 2020; 323: 130-139

Patienten, die einer Knie-TEP unterzogen werden, haben ein hohes Risiko für postoperative venöse Thromboembolien (VTE). Um dieses Risiko zu reduzieren, werden Antikoagulanzien eingesetzt wie Enoxaparin, der Faktor Xa und Thrombin inhibiert, oder Apixaban, welches nur Faktor Xa inhibiert. Obwohl diese Substanzen effektiv sind, sind sie mit einem Blutungsrisiko assoziiert, sodass weiterhin nach sichereren Antikoagulanzien gesucht wird.

Weitz und Kollegen aus East Hamilton, Kanada, verglichen in ihrer randomisierten Nichtunterlegenheitsstudie unterschiedliche Dosen von Osocimab mit Enoxaparin und Apixaban zur Thromboseprophylaxe bei Patienten nach Knie-TEP. Das primäre Outcome war die venöse Thromboembolie zwischen dem 10. und 13. postoperativen Tag. Eine 5\%-Nichtunterlegenheitsspanne wurde gewählt im Vergleich zu Enoxaparin.

\section{Ergebnisse}

813 Studienteilnehmer wurden eingeschlossen (mittleres Alter 66,5 Jahre; BMI 32,7; 74,2\% weiblich). 600 Personen wurden für die Primäranalyse in die Prä-Protokoll-Population aufgenommen. Unter den Patienten nach Knie-TEP erfüllten die Dosen von $0,6 \mathrm{mg} / \mathrm{kg}, 1,2 \mathrm{mg} / \mathrm{kg}$ und $1,8 \mathrm{mg} / \mathrm{kg}$ von Osocimab die Kriterien für die Nichtunterlegenheit im Vergleich zu Enoxaparin. Die präoperative Gabe von 1,8 mg/kg Osocimab war im Vergleich zu Enoxaparin für den primären Endpunkt der Inzidenz von VTE nach 10 und 13 Tagen postoperativ überlegen.

\section{FAZIT}

Die Autoren konnten feststellen, dass Osocimab dosisabhängig Enoxaparin nicht unterlegen war und in der präoperativen Gabe von 1,8 mg/kg Enoxa- parin hinsichtlich des primären Endpunktes überlegen war. Die Autoren nennen folgende Limitationen ihrer Studie: zum einen die Stichprobengröße, eine Verblindung hinsichtlich der Behandlungsallokation könnte die Entscheidung hinsichtlich der chirurgischen Intervention für das Blutungsmanagement beeinflusst haben; ferner wurden nur Patienten in Allgemeinanästhesie eingeschlossen, sodass Aussagen zur Spinalanästhesie nicht möglich sind. Weitere Studien, so die Autoren, sind notwendig, um die Effektivität und Sicherheit von Osocimab in dieser Indikation zu bestätigen.

MOR Dr. med. Benedikt Lampl, Regensburg 